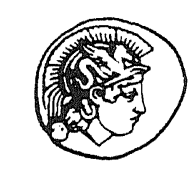

PERGAMON

Nonlinear Analysis $42(2000) 423-438$

Analysis

\title{
Spatial and time localization of solutions of the Boussinesq system with nonlinear thermal diffusion
}

\author{
G. Galiano* \\ Department of Modelling, Analysis and Simulation, CWI 1090 GB Amsterdam, Netherlands \\ Received 10 September 1997; accepted 15 July 1998
}

Keywords: Free convection; Free boundaries; Extinction in finite time; Energy method

\section{The model}

The Boussinesq system of hydrodynamics equations $[4,18]$, arises from a zero-order approximation to the coupling between the Navier-Stokes equations and the thermodynamic equation [17]. The presence of density gradients in a fluid leads to the conversion of gravitational potential energy into motion through the action of buoyant forces. Density differences are induced, for instance, by gradients of temperature arising by nonuniform heating of the fluid. In the Boussinesq approximation of a large class of flow problems thermodynamical coefficients such as viscosity, specific heat and thermal conductivity, can be assumed constant leading to a coupled system with linear secondorder operators in the Navier-Stokes and heat equations (see, e.g., [9-11, 15, 21]). However, there are some fluids like lubricants or some plasma flow for which this is no longer an accurate assumption (see, e.g. $[9,14,19]$ ). In this situation the following system of quasilinear equations must be considered [17]:

$$
\begin{aligned}
& \mathbf{u}_{t}+(\mathbf{u} \cdot \nabla) \mathbf{u}-\operatorname{div}(\mu(\theta) D(\mathbf{u}))+\nabla p=\mathbf{F}(\theta), \\
& \operatorname{div} \mathbf{u}=0, \\
& \mathscr{C}(\theta)_{t}+\mathbf{u} \cdot \nabla \mathscr{C}(\theta)-\Delta \varphi(\theta)=0,
\end{aligned}
$$

${ }^{*}$ Correspondence address: Dpto. Matematicas, Fac. Ciencias, Universidad de Oviedo, 33007 Oviedo, Spain.

E-mail address: galiano(a)anan.ciencias.uniovi.es (G. Galiano) 
where $\mathbf{u}$ is the velocity field of the fluid, $\theta$ the temperature, $p$ the pressure, $\mu(\theta)$ the viscosity of the fluid, $\mathbf{F}(\theta)$ the buoyancy force, $D(\mathbf{u}):=\nabla \mathbf{u}+\nabla \mathbf{u}^{\mathrm{T}}$,

$$
\mathscr{\zeta}(\theta):=\int_{\theta_{0}}^{\theta} C(s) \mathrm{d} s \text { and } \varphi(\theta):=\int_{\theta_{0}}^{\theta} \kappa(s) \mathrm{d} s,
$$

with $C(\theta)$ and $k(\theta)$ being the specific heat and thermal conductivity of the fluid, respectively. Assuming, as usual, $C>0$ then $\mathscr{E}$ is inversible, and so $\theta=\mathscr{C}^{-1}(\bar{\theta})$ for some real argument $\bar{\theta}$. Then we can define the functions

$$
\bar{\varphi}(\bar{\theta}):=\varphi \circ \mathscr{C}^{-1}(\bar{\theta}), \quad \overline{\mathbf{F}}(\bar{\theta}):=\mathbf{F} \circ \mathscr{C}^{-1}(\bar{\theta}), \quad \bar{\mu}(\bar{\theta}):=\mu \circ \mathscr{C}^{-1}(\bar{\theta}) .
$$

Substituting these expressions in Eq. (1) and omitting the bars we get the following formulation of the Boussinesq system:

$$
\begin{aligned}
& \mathbf{u}_{t}+(\mathbf{u} \cdot \nabla) \mathbf{u}-\operatorname{div}(\mu(\theta) D(\mathbf{u}))+\nabla p=\mathbf{F}(\theta), \\
& \operatorname{div} \mathbf{u}=0, \\
& ()_{t}+\mathbf{u} \cdot \nabla()-\Delta \varphi(\theta)=0 .
\end{aligned}
$$

We briefly comment on some interesting features that characterize this model. There are two paradigmatic situations: the fast and the slow heat diffusion. These cases mathematically correspond to the singular or degenerate character of the heat equation which may occur according to the relative behavior of $C$ and $k$. This was already pointed out in [6]. The main goal of this paper is to improve the results presented in [6]. To state our main results recall that since $C$ and $\kappa$ are nonnegative, their primitives $\mathscr{C}$ and $\varphi$ are nondecreasing functions. Suppose that a perturbation of a constant temperature $\theta_{0}$ causes a small gradient of temperature between the boundary (higher temperature) and the interior (lower temperature) in a neighborhood, and assume that the behavior of $\mathscr{C}$ and $\varphi$ near $\theta_{0}$ can be approximate as

$$
\mathscr{b}(s) \sim c_{1}\left(s-\theta_{0}\right)+c_{2}\left(s-\theta_{0}\right)^{p}, \quad \varphi(s) \sim k_{1}\left(s-\theta_{0}\right)+k_{2}\left(s-\theta_{0}\right)^{q}
$$

for $s>0_{0}$, with $p, q>0$. From Eq. (1) we have that

$$
\bar{\varphi}^{\prime}(\mathscr{C}(s))=\varphi^{\prime}(s)\left(\mathscr{C}^{-1}\right)^{\prime}(\mathscr{C}(s))=\frac{\varphi^{\prime}(s)}{\mathscr{C}^{\prime}(s)}=\frac{k_{1}+k_{2} q\left(s-\theta_{0}\right)^{q-1}}{c_{1}+c_{2} p\left(s-\theta_{0}\right)^{p-1}} .
$$

Therefore, when $s \rightarrow 0_{0}$ (and then $\mathscr{E}(s) \rightarrow 0$ ) we get one of the following behaviors of $\bar{\varphi}^{\prime}$ close:

(i) if $p, q>1$ then $\bar{\varphi}^{\prime}(0)=k_{1} / c_{1}$,

(ii) if $1>q>p$ either $q>1>p$ then $\lim _{\mathscr{G}(s) \rightarrow 0} \bar{\varphi}^{\prime}(\mathscr{C}(s))=0$,

(iii) if $p>1>q$ either $1>p>q$ then $\lim _{\mathscr{G}(s) \rightarrow 0} \bar{\varphi}^{\prime}(\mathscr{C}(s))=+\infty$.

In the first case both linear parts in the above decomposition of 4 , and $\varphi$ dominate: this case arises, for instance, when conductivity and specific heat are taken as constants, leading to the classical heat equation with a linear diffusion term. In the other two cases the nonlinear parts dominate and this leads to two different situations:

- If $p<q$ : The specific heat dominates over the conductivity, i.e., when temperature approaches $\theta_{0}$ the fluid stores more heat and this is worstly conducted. We shall 
prove that a front of temperature $\theta=\theta_{0}$ arises. This type of phenomenon is known as slow diffusion: heat spends a positive time to spread over the neighborhood.

- If $p>q$ : The opposite effect arises: the conductivity dominates over the specific heat. In this case the phenomenon is called fast diffusion. We shall prove that, in fact, $\theta=\theta_{0}$ in the whole domain when the time is large enough.

In [7] we studied the well posedness of system (2) together with suitable initial and boundary conditions under suitable assumptions on the data including the cases of degenerate and singular parabolicity of the heat equation. This enforced, for wellknown reasons, the introduction of a notion of weak solution for the heat equation. In which respect to the Navier-Stokes equations, we followed the usual variational approach, already introduced by Leray [16], based on the consideration of the notion of weak solution for the Navier-Stokes equations holding in divergence free functional spaces (denoted with the subindex $\sigma$ ). More precisely, we considered the following problem:

$$
\begin{aligned}
& \mathbf{u}_{t}+(\mathbf{u} \cdot \nabla) \mathbf{u}-\operatorname{div}(\mu(\theta) D(\mathbf{u}))=\mathbf{F}(\theta) \quad \text { in } Q_{T}:=\Omega \times(0, T), \\
& \theta_{t}+u \cdot \nabla \theta-\Delta \varphi(\theta)=0 \quad \text { in } Q_{T}, \\
& \mathbf{u}=0 \quad \text { and } \quad \varphi(\theta)=\varphi_{D} \quad \text { in } \Sigma_{T}:=\partial \Omega \times(0, T), \\
& \mathbf{u}(\mathbf{x}, 0)=\mathbf{u}_{0}(\mathbf{x}) \text { and } \theta(\mathbf{x}, 0)=\theta_{0}(\mathbf{x}) \quad \text { for } x \in \Omega,
\end{aligned}
$$

with $\Omega \subset \mathbb{B}^{N}(N=2,3)$ a bounded domain and $T$ arbitrarily fixed. Besides, we assumed the following regularity on the auxiliary data: $\mathbf{u}_{0} \in L_{\sigma}^{2}(\Omega), \theta_{0} \in L^{\infty}(\Omega), \theta_{0} \geq 0$ and $\varphi_{D} \in L^{2}\left(0, T ; H^{1}(\Omega)\right) \cap H^{1}\left(0, T ; L^{2}(\Omega)\right)$.

Definition (Weak solution). The pair $(\mathbf{u}, 0)$ is said to be a weak solution of Eq. (3) if:

(i)

$$
\begin{aligned}
& \mathbf{u} \in L^{2}\left(0, T ; W_{\sigma}^{1,2}(\Omega)\right) \cap L^{\infty}\left(0, T ; L_{\sigma}^{2}(\Omega)\right) \\
& \varphi(\theta) \in \varphi_{D}+L^{2}\left(0, T ; H_{0}^{1}(\Omega)\right) \text { and } \theta \in L^{\infty}\left(Q_{T}\right) .
\end{aligned}
$$

(ii) $\mathbf{u}(0)=\mathbf{u}_{0}$ and for any test function $\mathbf{w} \in W_{\sigma}^{1,2}(\Omega) \cap L_{\sigma}^{N}(\Omega)$ it holds

$$
\int_{\Omega}\left(\mathbf{u}_{t} \cdot \mathbf{w}+(\mathbf{u} \cdot \nabla) \mathbf{u} \cdot \mathbf{w}+\mu(\theta) D \mathbf{u}: \nabla \mathbf{w}\right)=\int_{\Omega} \mathbf{F}(\theta) \cdot \mathbf{w} \quad \text { a.e. } t \in(0, T),
$$

(iii) $\theta_{t} \in L^{2}\left(0, T ; H^{-1}(\Omega)\right)$ and for any test functions $\zeta \in L^{2}\left(0, T ; H_{0}^{1}(\Omega)\right)$ and $\psi \in L^{2}\left(0, T ; H_{0}^{1}(\Omega)\right) \cap W^{1,1}\left(0, T ; L^{2}(\Omega)\right)$, with $\psi(T)=0$, it holds

$$
\int_{0}^{T}\left\langle\theta_{t}, \zeta\right\rangle+\int_{0}^{T} \int_{\Omega}(\nabla \varphi(\theta)-(0 \mathbf{u}) \cdot \nabla \zeta=0
$$

and

$$
\int_{0}^{T}\left\langle 0_{t}, \psi\right\rangle+\int_{0}^{T} \int_{\Omega}\left(0-0_{0}\right) \psi_{t}=0
$$


In [7] we proved that under the assumptions

$$
\begin{aligned}
& \varphi \in \mathscr{C}\left([0, \infty) \cap \cap \mathscr{C}^{1}((0, \infty)), \quad \varphi(0)=0, \varphi\right. \text { nondecreasing, } \\
& \mathbf{F} \in \mathscr{C}_{\mathrm{loc}}^{0,1}\left([0, \infty) ; \mathbb{R}^{N}\right), \\
& \mu \in \mathscr{C}_{\mathrm{loc}}^{0,1}([0, \infty)) \text { and satisfies } 0<m_{0} \leq \mu(s) \leq m_{1} \quad \forall s \in[0, \infty) \\
& \text { and if } \mu^{\prime} \neq 0 \text { and } \mathbf{F}^{\prime} \neq 0 \text { then } \varphi^{-1} \text { is Hölder continuous of exponent } \alpha,
\end{aligned}
$$

there exists a weak solution of Eq. (3). Besides, if $N=2, \mu$ is constant and either $\varphi^{-1} \in \mathscr{C}_{\text {loc }}^{0,1}([0, \infty))$ or

$$
\varphi^{\prime}(0)=0, \quad \varphi^{\prime}(s)>0 \quad \text { and } \quad \varphi^{\prime \prime}(s)>0 \quad \text { if } s>0 \quad \text { and } \quad \nabla \theta \in L^{2}\left(Q_{T}\right)
$$

then the solution is unique. In this paper we shall show that, under suitable assumptions on the data, spatial or time localization of the temperature component of any weak solution occurs.

\section{Spatial localization}

In this section we present results concerning the existence and spatial localization of free boundaries (boundary of sets where $\{\theta=0\}$ ) for problem (3), more precisely, the finite speed of propagation and the waiting time properties (see Theorems 2.1 and 2.2 below). It is well known that solutions to porous medium type equations exhibit the property of finite speed of propagation (compactly supported solutions) when the initial data vanishes in some part of the domain. The usual way to show that this property holds relies in the existence of a comparison principle for the problem and the use of special subsolutions that already enjoy the property. However, this method is very sensible to perturbations in the problem and it fails in its application when subsolutions are hard to find (for instance, when coefficients depend on space and time variables or when space dimension is greater than one) or when, simply, a comparison principle does not hold (systems of equations, in general). In this situation more general methods (although less accurate) must be considered.

The method we use is based on a local energy method for free boundary problems (see $[1,8]$ ). The idea is to introduce an energy functional (usually given by the norms in the natural energy spaces associated to the problem) and to deduce a differential inequality for the energy functional. From this inequality the desired qualitative property of the solutions can be obtained. This method has two principal features. Firstly, it is a local method, i.e., it operates in subsets of the corresponding domain without need of global information like boundary conditions or boundedness of the domain. Secondly, it has a very general setting, allowing to consider, for instance, systems of equations in any space dimension or with coefficients depending on the space and time variables. This method does not need any monotonicity assumption on the nonlinearities and it requires no comparison principle to hold. In return, however, it only provides a qualitative insight into the problem but not valuable quantitative estimates. 
In this paper we introduce a variation of this energy method intended to handle the transport term present in the heat equation. Intuitively, this term should not affect the existence and properties of the free boundary but its location, moving it in the direction of the characteristics associated to the velocity field. This approach improves previous results when a transport term is present in the equation (see $[2,8]$ ) and can be regarded as a generalization of the method. It is also straightforward to apply to other properties like the shrinking of the support or the formation of dead cores (see [2,12]). Finally, it also facilitates the study of these properties in systems of equations coupled, among others, through transport terms (see [12]).

In this section we assume that $\varphi(s):=s^{m}$. Given a weak solution $(\mathbf{u}, \theta)$ of Eq. (3) we perform the change of unknown $\hat{\theta}:=\theta^{m}$. Writing $q:=1 / m$, introducing the expression of $\hat{\theta}$ in the second equation of Eq. (3) and omitting the hats we are led to the following form of the heat equation:

$$
\left(\theta^{q}\right)_{t}+\mathbf{u} \cdot \nabla \theta^{q}-\Delta \theta=0 .
$$

In order to define the (local) characteristics associated to $\mathbf{u}$ we must assume certain regularity on this vectorial field, namely $\mathbf{u} \in L^{\infty}\left(0, T ; \mathscr{C}^{0,1}(\bar{\Omega})\right)$. Since $\mathbf{u}=\mathbf{0}$ in $\partial \Omega$ we can extend $\mathbf{u}$ by zero to all $\mathbb{R}^{N}$ to get $\mathbf{u} \in L^{\infty}\left(0, T ; \mathscr{C}^{0,1}\left(\mathbb{R}^{N}\right)\right)$. It is well known that under this regularity, the vectorial function $\chi$ given as a solution of the problem

$$
\begin{aligned}
& \frac{\partial \chi}{\partial t}(\mathbf{x}, t)=\mathbf{u}(\chi(\mathbf{x}, t), t) \quad \text { in } \omega \times(0, T), \\
& \chi(\mathbf{x}, 0)=\mathbf{x} \quad \text { in } \omega,
\end{aligned}
$$

with $\omega \subset \mathbb{R}^{N}$, is uniquely defined with the regularity $\chi \in \mathscr{C}^{1}(\bar{\omega} \times(0, T))$.

In the following we shall suppose that the initial data $\theta_{0}$ vanishes in some ball $\mathbf{B}_{\rho_{0}}$ of radius $\rho_{0}$, centered in $\mathbf{x}_{0}$ and compactly imbedded in $\Omega$. Then the following property is a consequence of the continuity of $\chi$ and of $\chi(\cdot, 0) \equiv$ Identity:

there exist $\hat{t}$ and $\rho_{1}>\rho_{0}$ such that if $t<\hat{t}$ and $\rho<\rho_{1}$ then $\chi\left(\mathbf{B}_{\rho}, t\right) \subset \Omega$.

We first state the result on the finite speed of propagation and waiting time property along the characteristics:

Theorem 2.1. Suppose that $q<1$ and $\theta_{0} \equiv 0$ in $\mathbf{B}_{\rho_{0}}$. Then there exists a $t^{*} \in(0, \hat{t})$ and a continuous function $r(\tau)$ defined in $\left(0, t^{*}\right)$, with $r(0)=\rho_{0}$ such that any second component of a weak solution of Eq. (3) satisfies

$$
\theta(\mathbf{x}, t) \equiv 0 \quad \text { a.e. in }\left\{(\mathbf{x}, t): \mathbf{x} \in \chi\left(\mathbf{B}_{r(t)}, t\right), t \in\left(0, t^{*}\right)\right\} .
$$

Besides, if the initial data satisfies the following flatness condition:

$$
\int_{\mathbf{B}_{\rho}}\left|\theta_{0}\right|^{q+1} \mathrm{~d} \mathbf{x} \leq \delta_{0}\left(\rho-\rho_{0}\right)_{+}^{1 /(1-\kappa)} \quad \text { a.e. } \rho<\rho_{1}
$$

for some $\delta_{0}>0$ and with $\kappa<1$ given by Eq. (26) then there exists a $t_{*} \in(0, \hat{t})$ such that

$$
\theta(\mathbf{x}, t) \equiv 0 \quad \text { a.e. in }\left\{(\mathbf{x}, t): \mathbf{x} \in \chi\left(\mathbf{B}_{\rho_{0}}, t\right), t \in\left(0, t_{*}\right)\right\} .
$$


Let us briefly comment here some extensions of this result. First, with respect to the structural assumptions on the heat equation we only need to assume a structure of the type

$$
\left(|\theta|^{q-1} \theta\right)_{t}+\operatorname{div}\left(\mathbf{u}|\theta|^{q-1} \theta\right)-\operatorname{div} \mathbf{A}(x, t, \theta, \nabla \theta)+B(x, t, \theta)=f(x, t)
$$

with $\mathbf{u}$ and $\mathbf{A}$ vectorial fields and $B$ a scalar function satisfying

$$
\begin{aligned}
& M_{1}|\xi|^{r} \leq \mathbf{A}(\mathbf{x}, t, s, \xi) \cdot \xi \leq M_{2}|\xi|^{r} \quad \text { for } r>1 \\
& s B(\mathbf{x}, t, s) \geq m_{3}|s|^{p+1} \quad \text { for } p>0 \\
& \|\mathbf{u}(\mathbf{x}, t)\|_{L^{\infty}\left(Q_{T}\right)} \leq M_{4}, \\
& \|\operatorname{div} \mathbf{u}(\mathbf{x}, t)\|_{L^{\infty}\left(Q_{T}\right)} \leq M_{5} \quad \text { or } \operatorname{div}(\mathbf{u}(\mathbf{x}, t)) \geq 0,
\end{aligned}
$$

$\forall(\mathbf{x}, t, s, \xi) \in \Omega \times \mathbb{R}_{+} \times \mathbb{R} \times \mathbb{R}^{N}$ and for some positive constants $M_{i}$. In this situation, if $m_{3}=0$ (resp. $\left.m_{3}>0\right), q<r-1$ (resp. $\left.p<q \leq r-1\right)$ and $f(x, t)=0$ in $\chi\left(\mathbf{B}_{\rho_{0}} \times(0, T)\right.$ ) then the result on finite speed of propagation still holds. If, in addition, instead of Eq. (9) we assume

$$
\int_{\mathbf{B}_{\mu}}\left|\theta_{0}\right|^{q+1} \mathrm{~d} \mathbf{x}+\int_{\mathbf{B}_{\mu}}|f|^{q /(q+1)} \leq \delta_{0}\left(\rho-\rho_{0}\right)_{+}^{1 /(1-\kappa)} \text { a.e. } \rho<\rho_{1},
$$

then the result on existence of a waiting time also holds. The second kind of extension that can be performed is related to other properties of the free boundary, for instance, the formation of a dead core:

Suppose that $m_{3}>0$, and $f \equiv 0$. Assume that

$$
p<q \leq r-1 \text { and } 1+p \leq q \frac{r}{r-1} .
$$

Then if the global energy is small enough there exist constants $t^{*} \in(0, t)$ and $v>0$ such that any second component of a weak solution of Eq. (3) satisfies

$$
\begin{aligned}
\theta(\mathbf{x}, t) & \equiv 0 \quad \text { a.e. in }\left\{(\mathbf{x}, t): \mathbf{x} \in \chi\left(\mathbf{B}_{r_{(\tau)}}, \tau\right), \tau \in\left(t^{*}, T\right)\right\}, \\
\text { with } r(\tau) & :=\left(\tau-t^{*}\right)^{v} .
\end{aligned}
$$

The proofs of these results follow the line of [2] in combination with the proof of Theorem 2.1 (see [12] for more details).

Finally, we state a result on finite speed of propagation for which weaker requirements on the regularity of the velocity field are needed (see [13]). More precisely, we shall assume only that $\mathbf{u} \in L_{\sigma}^{\infty}\left(Q_{T}\right)$. Although in this case we cannot properly define 
the characteristics associated to $\mathbf{u}$, the boundedness of the velocity field still allows us to use similar ideas than above.

Theorem 2.2. Suppose that $q<1$ and that $\theta_{0} \equiv 0$ in $\mathbf{B}_{\mu_{0}}$. Then there exist a $t^{*}>0$ and a nonnegative function $r(\tau)$ defined in $\left(0, t^{*}\right)$, with $r(0)=\rho_{0}$, such that any second component of a weak solution of Eq. (3) satisfies

$$
\theta(\mathbf{x}, t) \equiv 0 \quad \text { a.e. in }\left\{(\mathbf{x}, t): \mathbf{x} \in \mathbf{B}_{r(t)}, t \in\left(0, t^{*}\right)\right\} \text {. }
$$

Theorems 2.1 and 2.2 improve previous results in the literature of scalar differential equations when the transport term $\mathbf{u}$ is nontrivial (see $[2,6,8])$. Moreover, as far as we know, properties like the formation of a dead core in systems or scalar equations containing a transport term do not seem to have been treated till the date.

\subsection{Proofs of Theorems 2.1 and 2.2}

In the proof of both theorems we shall use special test functions to localize the natural energies associated to the problem in suitable sets of the form

$$
\mathscr{P} \equiv \mathscr{P}(\rho, t):=\left\{(\mathbf{x}, \tau) \in \mathbb{R}^{N} \times \mathbb{R}_{+}: \mathbf{x} \in \chi\left(\mathbf{B}_{R}, \tau\right), \tau \in(0, t)\right\}
$$

with $\mathbf{B}_{R}$ the ball of $\mathbb{R}^{N}$ centered in the origin and of radius $R \equiv R(\rho, \tau)$, for certain nonnegative arguments $\rho, \tau$, and with $\chi(\cdot, \tau)$ given by Eq. (7). We introduce the temporal sections of

$$
P(s):=\mathscr{P} \cap\{\tau=s\} \subset \mathbb{R}^{N}, \quad s \in(0, t),
$$

so $\mathscr{P}=\bigcup_{s \in(0, t)} P(s)$, and the lateral boundary of $\mathscr{P}$

$$
\partial_{1} \mathscr{P}:=\left\{(\mathbf{x}, \tau) \in \mathbb{R}^{N} \times \mathbb{R}_{+}: \mathbf{x} \in \partial \chi\left(\mathbf{B}_{R}, \tau\right), \quad \tau \in(0, t)\right\}
$$

so the parabolic boundary of $\mathscr{P}$ is given by $\partial \mathscr{P}=P(0) \cup P(t) \cup \partial_{1} P$. We recall here some basic properties related with this collection of sets (see, e.g., [12]). If $\left(n_{t}, \mathbf{n}_{x}\right)$ is the unitary outward normal vector to $\mathscr{P}$ then it holds

$$
n_{t}+\mathbf{w} \cdot \mathbf{n}_{x}=-\frac{\partial R}{\partial \tau} \operatorname{det} \frac{D \chi}{D \mathbf{x}}
$$

and for any integrable function $g$ it holds

$$
\frac{\mathrm{d}}{\mathrm{d} \rho} \int_{\mathscr{\rho}(\rho, t)} g(\mathbf{x}, \tau) \mathrm{d} \mathbf{x} \mathrm{d} \tau=\int_{\eta_{1, \rho} \rho(\rho, t)} \frac{\mathrm{d} R}{\mathrm{~d} \rho} g(\mathbf{x}, \tau) \mathrm{d} \mathbf{x} \mathrm{d} \tau,
$$

where we used that being $\chi(\cdot, \tau)$ a diffeomorphism we have $\partial \chi\left(\mathbf{B}_{R}, \tau\right)=\chi\left(\partial \mathbf{B}_{R}, \tau\right)$. In this domain we consider the local energy functions defined by

$$
E(\rho, t):=\int_{, \rho}|\nabla \theta(\mathbf{x}, \tau)|^{2} \quad \text { and } \quad b(\rho, t):=\text { ess } \sup _{\tau \in(0, t)} \int_{P(\tau)}|\theta(\mathbf{x}, \tau)|^{q+1} .
$$


Next, we shall show how the local energies come into our problem. If $\theta$ is the second component of any weak solution of Eq. (3) we choose the following test function in Eqs. (5) and (6):

$$
\begin{aligned}
& \xi_{\mathscr{P}}(\theta(\mathbf{x}, t)):=\theta(\mathbf{x}, t) \quad \text { if }(\mathbf{x}, t) \in \mathscr{P}, \\
& \xi_{\mathscr{P}}(\theta(\mathbf{x}, t)):=0 \text { otherwise. }
\end{aligned}
$$

In [8] it is proven by truncature and regularization techniques that $\xi_{. p}$ is an admissible test function. After applying the divergence theorem we get

$$
\frac{q}{q+1} \int_{\partial \mathscr{P}}\left(\theta^{q+1} n_{t}+\theta^{q+1} \mathbf{u} \cdot \mathbf{n}_{x}\right) \mathrm{d} \mathbf{x} \mathrm{d} \tau+\int_{\mathscr{P}}|\nabla \theta|^{2} \mathrm{~d} \mathbf{x} \mathrm{d} \tau=\int_{\partial, \mathcal{P}} \theta \nabla \theta \cdot \mathbf{n}_{x} \mathrm{~d} \mathbf{x} \mathrm{d} \tau .
$$

Using the decomposition $\partial \mathscr{P}=P(0) \cup P(t) \cup \partial_{l} \mathscr{P}$ and taking into account that $n_{t}=1$ and $\mathbf{n}_{x}=0$ in $P(t)$ and that $n_{t}=-1$ and $\mathbf{n}_{x}=0$ in $P(0)$, we obtain

$$
\begin{aligned}
& \frac{q}{q+1} \int_{P(t)}|\theta(t)|^{q+1} \mathrm{~d} \mathbf{x}+\int_{\mathscr{P}}|\nabla \theta|^{2} \mathrm{~d} \mathbf{x} \mathrm{d} \tau \\
& \leqslant \int_{\partial_{l} \mathscr{P}}|\nabla \theta||\theta|\left|\mathbf{n}_{x}\right| \mathrm{d} \mathbf{x} \mathrm{d} \tau-\frac{q}{q+1} \int_{\partial_{l}, P}|\theta|^{q+1}\left(n_{t}+\mathbf{w} \cdot \mathbf{n}_{x}\right) \mathrm{d} \mathbf{x} \mathrm{d} \tau \\
& \quad+\frac{q}{q+1} \int_{P(0)}\left|\theta_{0}\right|^{q+1} \mathrm{~d} \mathbf{x}:=j_{1}+j_{2}+j_{3} .
\end{aligned}
$$

The proofs of the theorems share a common scheme: firstly we perform estimates of the terms $j_{i}$ of Eq. (16) by using well-known inequalities, like Hölder, Young and Poincaré's, together with the use of an interpolation-trace inequality (see [8]) that allows us to deduce a differential inequality for the local energy $E$. Secondly, a direct integration of such an inequality leads to the desired result.

We start with the proof of Theorem 2.2. Notice that for this result we do not need to consider the characteristics associated to $\mathbf{u}$ and therefore $\chi$ must be regarded as the identity.

Proof of Theorem 2.2. We proceed in two steps:

Step 1. We consider the domain of integration in Eq. (16) as the collection of truncated cones given by

$$
\mathscr{P}(\rho, t):=\left\{(\mathbf{x}, \tau): \mathbf{x} \in \mathbf{B}_{R}, \tau \in(0, t)\right\},
$$

with $R \equiv R(\rho, \tau):=\rho-\tau u, u:=\|\mathbf{u}\|_{L^{\infty}\left(Q_{T}\right)}, \rho \in I:=\left(t u+\varepsilon, \rho_{0}\right]$ and $t<t_{1}:=\left(\rho_{0}-\varepsilon\right) / u$. Notice that $I$ is nonempty since, by construction, $t u+\varepsilon<\rho_{0}$. We begin by estimating the term $j_{1}$. On one hand, the function $\rho \rightarrow \int_{\partial_{1}: \rho}|\nabla \theta|^{2}$ is well defined for a.e. $\rho \in I$ because of the regularity $\theta \in L^{2}\left(0, T ; H^{1}(\Omega)\right)$. We can use Hölder's inequality to get

$$
j_{1} \leq\left(\int_{\partial_{1}: \mathscr{P}}|\theta|^{2}\right)^{1 / 2}\left(\int_{\partial_{1}, \mathscr{P}}|\nabla \theta|^{2}\right)^{1 / 2} .
$$



On the other hand, by Eq. (14) we have $i E C \rho=\int_{i},|\Gamma|^{2}$ a.e. $\rho \in I$, and from
Eq. (17) we get

$$
j_{1} \leq\|\theta\|_{L i(i, \varphi(t))}\left(\frac{\partial E}{\partial \rho}(\rho, t)\right)^{12} .
$$

Taking into account that $r l, P=\bigcup_{\tau \in(0, t)} i P(\tau)$, with $i P(\tau) \equiv\left\langle\mathbf{B}_{R}\right.$, and applying the interpolation-trace inequality (see $[8]$ ) we obtain

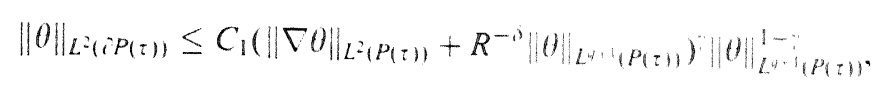

with $C_{1}>0$ an universal constant and with

$$
\gamma:=\frac{2 N-(q+1)(N-1)}{2(N+q+1)-N(q+1)} \in(0,1) \text { and } \delta:=1+\frac{N(1-q)}{2(q+1)} .
$$

Notice that since $\rho \in I$

$$
R(\tau)^{-\dot{\delta}} \leq\left(\min _{\tau \in(0 . t)}(\rho-u \tau)\right)^{-j} \leq \varepsilon^{-i}
$$

Defining $L:=C_{1} \max \left\{1, \varepsilon^{-\gamma \dot{ }}\right\}$ and integrating expression $(19)$ in $(0, t)$ we obtain

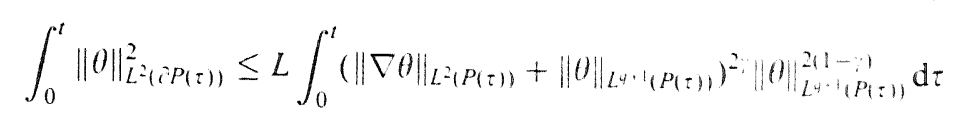

and thanks to the inequality $(a+b)^{2} \leq 2\left(a^{2}+b^{2}\right)$ and to Hölder's inequality with exponent $1 / \gamma$ we obtain

$$
\int_{0}^{t}\|\theta\|_{L^{2}(i P(\tau))}^{2} \leq 4 L^{2}\left(\int_{0}^{t}\|\nabla \theta\|_{L^{2}(P(\tau))}^{2}+\|\theta\|_{\left.L^{4} \cdot 1, P(\tau)\right)}^{2}\right)^{\prime}\left(\int_{0}^{t}\|\theta\|_{L^{4+1}(P(\tau))}^{2}\right)^{1-\gamma} .
$$

Using that $q<1$ and that $b$ is nondecreasing with respect to $\rho$, and substituting the expressions of energies given in Eq. (15) we obtain

$$
\begin{aligned}
& \left(\int_{0}^{t}\|\theta\|_{L^{\prime}(i P(\tau)}^{1 / 2}\right)^{1 / 2} \\
& \quad \leq 2 L t^{(1-i) \cdot 2}\left(E(\rho, t)+t b\left(\rho_{0}, t\right)^{2(q+1)-1} b(\rho, t)\right)^{: 2} b(\rho, t)^{(1-i)(q+1),}
\end{aligned}
$$

and therefore, defining

$$
K_{0}(t):=2 L t^{(1-i) 2} \max \left\{1, t_{1} b\left(\rho_{0}, t_{1}\right)^{2(q+1)-1}\right\},
$$

we get

$$
\left(\int_{0}^{t}\|\theta\|_{L^{2}(\hat{C} P(\tau))}^{2}\right)^{1 / 2} \leq K_{0}(t)(b(\rho, t)+E(\rho, t))^{r}
$$


with

$$
v:=\frac{\gamma}{2}+\frac{1-\gamma}{q+1}<1
$$

We conclude from Eq. (17) that

$$
j_{1} \leq K_{0}(t)\left(\frac{\partial E(\rho, t)}{\partial \rho}\right)^{1 / 2}(b(\rho, t)+E(\rho, t))^{v} .
$$

Finally, by assumption, $j_{3}=0$ and the estimate of $j_{2}$ is simple because the choice of the domain of integration. Indeed we have by Eq. (13) (remind that $\chi=$ identity) that

$$
n_{t}+\mathbf{u} \cdot \mathbf{n}_{x}=\|\mathbf{u}\|_{L^{\infty}}+\mathbf{u} \cdot \mathbf{n}_{x} \geq 0
$$

and therefore $j_{2} \leq 0$.

Step 2. We get from Eq. (16) that

$$
2 M\left(\int_{P(t)}|\theta|^{q+1}+E(\rho, t)\right) \leq K_{0}(t)\left(\frac{\partial E(\rho, t)}{\partial \rho}\right)^{1 / 2}(b(\rho, t)+E(\rho, t))^{v}
$$

with $M=q / 2(q+1)$. Since functions $E, b$ and $\partial E / \partial \rho$ are nondecreasing in time Eq. (24) remains valid if we change $\int_{P(t)}|\theta|^{q+1}$ by $b(\rho, t)$ and $2 M$ by $M$. We get

$$
M(b(\rho, t)+E(\rho, t)) \leq K_{0}(t)\left(\frac{\partial E(\rho, t)}{\partial \rho}\right)^{1 / 2}(b(\rho, t)+E(\rho, t))^{v}
$$

and therefore

$$
M^{2}(b(\rho, t)+E(\rho, t))^{\kappa} \leq K^{2}(t) \frac{\partial E(\rho, t)}{\partial \rho} \text { a.e. } \rho \in I,
$$

with

$$
\kappa:=2(1-v)
$$

$v$ given by Eq. (22) and

$$
K(t):=\frac{K_{0}(t)}{M}=\frac{C_{1}}{M} \max \left\{1, \varepsilon^{\gamma \delta}\right\}^{1 / 2} \max \left\{1, t_{1} M^{2 /(q+1)-1}\right\} t^{(1-\gamma) / 2} .
$$

Due to the crucial assumption $q<1$ we have that $\kappa<1$ and a direct integration of Eq. (25) in $\left(\rho, \rho_{0}\right)$ with $\rho \in I$ leads to

$$
E^{1-\kappa}(\rho, t) \leq E^{1-\kappa}\left(\rho_{0}, t\right)-K^{-2}(t)\left(\rho_{0}-\rho\right)
$$

We define

$$
z(t):=\rho_{0}-K^{2}(t) E^{1-\kappa}\left(\rho_{0}, t\right), \quad t>0 .
$$

Notice that $z(0)=\rho_{0}$ because $\lim _{t \rightarrow 0} E^{1-\kappa}\left(\rho_{0}, t\right) t^{1-\gamma}=0$ (here we used the definition of $K$ given in Eq. (27) and that $z(t)$ is continuous and decreasing with $\lim _{t \rightarrow \infty} z(t)=$ 
$-\infty)$. Therefore $z(t)$ has an unique positive zero that we denote by $t_{0}$. With this definition of $z(t)$ we have that if $t \in\left(0, t^{*}\right)$ for some $t^{*} \in\left(0, \min \left\{\hat{t}, t_{0}\right\}\right]$ and $\rho \in(u t+$ $\varepsilon, z(t))$ then Eq. (28) implies that $E(\rho, t) \equiv 0$. Notice that the interval $(u t+\varepsilon, z(t))$ is nonempty if

$$
\rho_{0}>K^{2}(t) E^{1-\kappa}\left(\rho_{0}, t\right)+u t+\varepsilon
$$

that we can ensure by taking $t^{*}, \varepsilon$ small enough. Finally, from the definition of $E(\rho, t)$ we deduce that $\theta(\mathbf{x}, t) \equiv 0$ if $|\mathbf{x}| \leq \rho-u t \leq z(t)-u t:=r(t)$, with $r(0)=\rho_{0}$ and $r(t)>0$ in $\left(0, t^{*}\right)$.

Proof of Theorem 2.1. The proofs of both assertions of this theorem are very like the previous proof, but taking advantage of the possibility of defining the characteristics associated to $\mathbf{u}$. We shall only prove the waiting time property being the proof of the finite speed of propagation a straightforward modification of the former.

Step 1. We consider the domain of integration given by

$$
\mathscr{P} \equiv \mathscr{P}(\rho, t):=\left\{(\mathbf{x}, \tau): \mathbf{x} \in \chi\left(B_{R}, \tau\right), \tau \in(0, t)\right\},
$$

with $\chi$ defined as the unique solution of Eq. $(7), R(\tau) \equiv \rho$ with $\rho \in\left(\rho_{0}, \rho_{1}\right)$ and $t \in(0, \hat{t})$ with $\hat{t}$ given by Eq. (8). Using Eq. (14) and Hölder's inequality we can estimate $j_{1}$ as in Eq. (17):

$$
\begin{aligned}
j_{1} & \leq \int_{\partial_{l} \mathscr{P}}|u||\nabla u|\left|\mathbf{n}_{x}\right| \leq\left(\int_{\partial_{l} \mathscr{P}}|\nabla u|^{2}\right)^{1 / 2}\left(\int_{\partial_{l} \mathscr{P}}|u|^{2}\left|\mathbf{n}_{x}\right|^{2}\right)^{1 / 2} \\
& \leq\left(\frac{\partial E}{\partial \rho}\right)^{1 / 2}\left(\int_{\partial_{l} \mathscr{P}}|u|^{2}\right)^{1 / 2},
\end{aligned}
$$

where we used that $\left|\mathbf{n}_{x}\right| \leq 1$. Since $\chi$ is a diffeomorphism, the interpolation-trace inequality remains valid for the same exponents $\gamma$ and $\delta$ although with, in general, a different universal constant $C_{1}$ depending on the Jacobian determinant of $\chi$ (see [12]):

$$
\|\theta\|_{L^{2}(\partial P(\tau))} \leq C_{1}\left(\|\nabla \theta\|_{L^{2}(P(\tau))}+\rho^{-\delta}\|\theta\|_{L^{q+1}(P(\tau))}\right)^{\gamma}\|\theta\|_{L^{q+1}(P(\tau))}^{1-\gamma} .
$$

Now, in the same way as in the previous proof we arrive at the estimate

$$
j_{1} \leq K_{0}(\rho, t)\left(\frac{\partial E}{\partial \rho}(\rho, t)\right)^{1 / 2}(b(\rho, t)+E(\rho, t))^{\nu},
$$

with $v$ given by Eq. (22) and $K_{0}$ given by Eq. (21) but with $L$ replaced by $L(\rho):=$ $C_{1} \max \left\{1, \rho^{-\gamma \delta}\right\}$. Applying Young's inequality with exponent $1 / v$ to Eq. (31) we have that for all $\varepsilon>0$

$$
j_{1} \leq C_{\varepsilon} K_{0}^{1 /(1-v)}\left(\frac{\partial E(\rho, t)}{\partial \rho}\right)^{1 / \kappa}+\varepsilon(b(\rho, t)+E(\rho, t)),
$$


with $C_{\varepsilon}=\varepsilon^{v /(v-1)}$ and $\kappa:=2(1-v)$. The estimate of $j_{2}$ follows directly from Eq. (13) and the choice of the domain of integration (involving a transformation along the characteristics defined by $\mathbf{u}$ ). We have

$$
n_{t}+\mathbf{u} \cdot \mathbf{n}_{x}=-\frac{\mathrm{d} R}{\mathrm{~d} \tau} \operatorname{det} \frac{D \chi}{D \mathbf{x}} \equiv 0,
$$

because $R$ does not depend on $\tau$. Therefore $j_{2} \equiv 0$. Finally, by assumption, $j_{3} \leq \delta_{0}(\rho-$ $\left.\rho_{0}\right)_{+}^{1 /(1-\kappa)}$

Step 2. As in step 2 of the previous proof, we take $M=q / 2(q+1)$ and find from Eq. (16) that

$$
\begin{aligned}
\frac{M}{2}(b(\rho, t)+E(\rho, t)) \leq & C_{\varepsilon} K_{0}^{1 / 1-v}(\rho, t)\left(\frac{\partial E(\rho, t)}{\partial \rho}\right)^{1 / k} \\
& +\varepsilon(b(\rho, t)+E(\rho, t))+\delta_{0}\left(\rho-\rho_{0}\right)_{+}^{1 /(1-\kappa)},
\end{aligned}
$$

with $\rho \in\left(\rho_{0}, \rho_{1}\right)$ and $t \in(0, \hat{t})$. Taking $\varepsilon<M / 4$ and rising both sides of Eq. (33) to the power $\kappa$ leads to

$$
E(\rho, t)^{\kappa} \leq \hat{C}_{1}(t) \frac{\partial E(\rho, t)}{\partial \rho}+\hat{C}_{2}\left(\rho-\rho_{0}\right)_{+}^{\kappa /(1-\kappa)}, \quad \rho \in\left(\rho_{0}, \rho_{1}\right), t \in(0, \hat{t}),
$$

with

$$
\hat{C}_{1}(t):=c\left(\frac{4 C_{k}}{M} \max _{\rho \in\left(\rho_{1}, p_{1}\right)} K_{0}^{1 / 1-\gamma}(\rho, t)\right)^{\kappa}=: \tilde{c} t^{1-\gamma}, \quad \hat{C}_{2}:=c\left(\frac{4 C_{\varepsilon} \delta_{0}}{M}\right)^{\kappa}
$$

and $c$ the constant of the inequality $(a+b)^{k} \leq c\left(a^{k}+b^{\kappa}\right)$. Notice that we again have the equivalency $q<1 \Leftrightarrow \kappa<1$. To conclude we construct an upper bound for $E$ that will imply the result. We consider the problem

$$
\begin{aligned}
& z^{\kappa}(\rho)=\tilde{c} t_{*}^{1-\gamma} \frac{\mathrm{d} z}{\mathrm{~d} \rho}(\rho)+\hat{C}_{2}\left(\rho-\rho_{0}\right)_{+}^{\kappa /(1-\kappa)} \text { in } \rho \in\left(\rho_{0}, \rho_{1}\right), \\
& z\left(\rho_{1}\right) \geq E\left(\rho_{1}, t_{*}\right),
\end{aligned}
$$

where $t_{*}$ is still to be chosen. The function $z(\rho):=A\left(\rho-\rho_{0}\right)_{+}^{1 /(1-\kappa)}$ solves the problem if we choose the positive constant $A$ such that

$$
A^{\kappa}=\frac{\tilde{c} t_{*}^{1-\gamma}}{1-\kappa} A+\hat{C}_{2} \quad \text { and } \quad A \geq E\left(\rho_{1}, t_{*}\right)\left(\rho_{1}-\rho_{0}\right)^{-1 /(1-\kappa)} .
$$

It is not difficult to check the existence of such a constant $A$ fulfilling Eq. (35) for $t_{*}$ small enough when we impose the additional restriction $A^{\kappa}>\hat{C}_{2}$. By the monotonicity of the problem we conclude that $E(\rho, t) \leq z(\rho)$ in $\rho \in\left(\rho_{0}, \rho_{1}\right), t \in\left(0, t_{*}\right)$ and therefore $E\left(\rho_{0}, t\right)=z\left(\rho_{0}\right)=0$ in $t \in\left(0, t_{*}\right)$, from where the assertion follows. 


\section{Time localization}

The property of time localization or extinction in finite time in evolution problems is associated to parabolic singularity and it may appear both in linear and nonlinear problems (see, e.g. [5] and the references therein). The techniques to study this property are similar to those used to prove the existence of free boundaries in parabolic degenerated equations, being the method of comparison the most successful because of the accuracy in the information obtained. As we already commented, certain kind of problems with general coefficients or in which the comparison principle fails to hold are no more tractable with this technique and other more general methods, like energy methods, have to be applied. We introduce more precisely the notion of extinction of finite time for problem (3) we shall deal with:

Definition 3.1. Given a weak solution $(\mathbf{u}, \theta)$ of Eq. (3), we say that $\theta$ has the extinction in finite time property if there exists an instant $t_{f}>0$ such that $\theta(\cdot, t)=0$ a.e. in $\Omega$ for all $t \geq t_{f}$.

In problem (3) extinction in finite time occurs when the heat conduction is fast, that is related to the class of functions $\varphi$ with global Lipschitz continuous inverse (and other additional properties), and it is a global property of solutions, i.e., a property that involves the structure of the differential equation as well as the behavior of the auxiliary conditions that solutions satisfy.

In the previous section we showed that the properties of localization in space of the support of solutions of Eq. (3) are local and therefore independent of the auxiliary data and of the boundedness or unboundedness of the domain. On the contrary, since the property of localization in time is global, it could be expected that the behavior of solutions is different when the domain under consideration is bounded or unbounded. And this is the case. For instance, for equations of the type

$$
\theta_{t}-\Delta \varphi(\theta)=0 \quad \text { in } \mathbb{R}^{N} \times(0, \infty)
$$

with $\varphi(s):=|s|^{m} \operatorname{sign}(s)$, the property holds if $0<m<(N-2) / N$ and $N \geq 3$ with the initial data in $L^{x+1}\left(\mathbb{R}^{N}\right) \cap L^{1}\left(\mathbb{R}^{N}\right)$ for some suitable $\alpha$ depending on $m$ [3]. However, this same equation in a bounded domain has the extinction property if $0<m<1$. More precisely, a sufficient condition for the occurrence of this property in solutions of Eq. (3) (and therefore for Eq. (36) when $\mathbf{u}=\mathbf{0}$ ) is given in the following theorem.

Theorem 3.1. Let $(\mathbf{u}, \theta)$ be a weak solution of problem (3) with boundary data satisfying $\varphi_{D} \equiv 0$ a.e. on $\Sigma_{T}$. Assume that

$$
\varphi^{\prime}(s) \geq c s^{m-1} \text { with } m \in(0,1)
$$

and $c>0$. Then $\theta$ has the extinction in finite time property. 
Proof. The proof follows closely the one given in [3]. The idea is to use $\theta^{p}$, for a suitable $p$, as a test function for the second equation of problem (3). Doing this formally we obtain

$$
\frac{\mathrm{d}}{\mathrm{d} t} \int_{\Omega} \theta^{p+1}+\int_{\Omega} \mathbf{u} \cdot \nabla \theta^{p+1}+\frac{p+1}{p} \int_{\Omega} \nabla \varphi(\theta) \cdot \nabla \theta^{p}=0,
$$

and by Eq. (37) and the fact that the velocity field is free divergence we get

$$
\frac{\mathrm{d}}{\mathrm{d} t} \int_{\Omega} \theta^{p+1}+\frac{4 c p}{p+1} \int_{\Omega}\left|\nabla \theta^{(p+m) / 2}\right|^{2} \leq 0 .
$$

Sobolev's theorem implies

$$
\int_{\Omega}\left|\nabla \theta^{(p+m) / 2}\right|^{2}=\left\|\theta^{(p+m) / 2}\right\|_{H_{0}^{\prime}(\Omega)}^{2} \geq C(q, \Omega)\left\|\theta^{(p+m) / 2}\right\|_{L^{4}(\Omega)}^{2},
$$

with $q \in\left[1,2^{*}\right]$, and $2^{*}$ the critical Sobolev's exponent of the imbedding $H^{1}(\Omega) \subset$ $L^{2 *}(\Omega)$. When $q=2(p+1) /(p+m)$ we have

$$
\int_{\Omega}\left|\nabla \theta^{(p+m) / 2}\right|^{2} \geq C(q, \Omega)\|\theta(t)\|_{L^{p+1}(\Omega)}^{p+m} .
$$

$q \in\left[1,2^{*}\right]$ holds if $2(p+1) / 2^{*}-p \leq m \leq p+2$. Since $m<1$, the upper bound is trivially satisfied meanwhile the lower bound holds whenever we take $p \geq\left(2-2^{*} m\right) /\left(2^{*}-2\right)$. Notice that in the case of $\Omega:=\mathbb{R}^{N}$ Sobolev's theorem is only valid for $q=2^{*}$ forcing to take $p=\left(2-2^{*} m\right) /\left(2^{*}-2\right)$ and therefore limiting the range of $m$ to that given in [3]. Gathering Eqs. (39) and (40) we deduce

$$
\frac{\mathrm{d}}{\mathrm{d} t}\|\theta(t)\|_{L^{p+1}(\Omega)}^{p+1}+C\|\theta(t)\|_{L^{p+1}(\Omega)}^{p+m} \leq 0
$$

with $C:=4 c p C(q, \Omega) /(p+1)$. Defining $E(t):=\|\theta(t)\|_{L^{p+1}(\Omega)}^{p+1}$ and $\alpha=(p+m) /(p+1)$, we formulate the above inequality into the problem

$$
\begin{aligned}
& \frac{\mathrm{d} E}{\mathrm{~d} t}(t)+C E(t)^{\alpha} \leq 0, \\
& E(0)=\left\|0_{0}\right\|_{L^{p+1}(\Omega)}^{p+1}:=E_{0} \geq 0,
\end{aligned}
$$

and, because of the crucial assumption $m<1$ we have that $\alpha<1$. The solution of the differential equation associated to Eq. (41) is an upper bound for any solution of the differential inequality. A direct integration shows that any solution of Eq. (41) must therefore satisfy

$$
E(t) \leq\left(E_{0}^{1-\alpha}-C(1-\alpha) t\right)_{+}^{1 /(1-\alpha)},
$$

and then $E(t)=0$ for $t \geq t_{f}:=E_{0}^{1-\alpha} / C(1-\alpha)$, from where the assertion follows.

This formal proof can be made rigorous, as in [3], by using techniques of semigroup theory. We first approximate $\mathbf{u} \in L^{\infty}\left(0, T ; L_{\sigma}^{2}(\Omega)\right) \cap L^{2}\left(0, T ; W_{\sigma}^{1,2}(\Omega)\right)$ by a sequence $\mathbf{u}_{n} \in \mathscr{C}^{\infty}\left(0, T ; \mathscr{C}_{\sigma}^{\infty}(\Omega)\right)$ and consider the equation

$$
0_{t}+\mathbf{u}_{n} \cdot \nabla \theta-\Delta \varphi(\theta)=0 \text { in } Q_{T},
$$


with $\varphi(\theta)=0$ in $\Sigma_{T}$ and $\theta(x, 0)=\theta_{0}(x) \in L^{\infty}(\Omega)$. We introduce the iteration $v_{0}:=\theta_{0}$ and $v_{i+1} \in L^{\infty}(\Omega)$ given by

$$
\frac{v_{i+1}-v_{i}}{\lambda}+\mathbf{u}_{n, i+1} \cdot \nabla v_{i+1}-\Delta \varphi\left(v_{i+1}\right)=0,
$$

with $\lambda>0$ and $\mathbf{u}_{n, i+1}(x):=\mathbf{u}_{n}(x, \lambda(i+1))$. Multiplying Eq. (43) by $v_{i+1}^{p}$, using that $v_{i} v_{i+1}^{p} \leq(1 /(p+1))\left(v_{i}^{p+1}-p v_{i+1}^{p+1}\right)$ and integrating on $\Omega$ yields

$$
\frac{1}{p+1} \frac{1}{\lambda} \int_{\Omega}\left(v_{i+1}^{p+1}-v_{i}^{p+1}\right)+C\left(\int_{\Omega} v_{i+1}^{p+1}\right)^{(p+m) /(p+1)} \leq 0
$$

where we used Sobolev's theorem and that $\operatorname{div} \mathbf{u}_{n, i+1}=0$. Defining $E(t):=\int_{\Omega} \theta^{p+1}(t)$, with $\theta$ the semigroup solution of Eq. (42) (that does exist, see. e.g. [20]), we deduce like in [3] that when $i \rightarrow \infty$ and $\lambda \rightarrow 0$ Eq. (44) implies

$$
-\int_{0}^{T} E(t) \psi^{\prime}(t)+c \int_{0}^{T} E(t)^{\alpha} \psi(t) \leq 0 \text { for all } \psi \in C_{0}^{\infty}(0, T),
$$

with $\alpha:=(p+m) /(p+1)$, from where Eq. (41) follows in the sense of distributions. Finally, since Eqs. (44) and (45) are independent of $\mathbf{u}_{n}$ a density argument allows us to conclude.

\section{Acknowledgements}

I cordially thank Jesús Ildefonso Díaz for his valuable suggestions to tackle the problems within this paper.

\section{References}

[1] S.N. Antontsev, On the localization of solutions of nonlinear degenerate elliptic and parabolic equations. Dokl. Akad. Nauk. SSSR 260 (6) (1981) 1289-1293 (in Russian) (English translation in Sov. Math. Dokl. 24 (2) (1981) 420-424).

[2] S.N. Antontsev, J.I. Diaz, S.I. Shmarev, The support shrinking properties for local solutions of quasilinear parabolic equations with strong absorption terms, Ann. Fac. Sci. Toulouse 4 (1) (1995) 5-30.

[3] P. Benilan, M.G. Crandall, The continuous dependence on $\varphi$ of solutions of $u_{t}-\Delta \varphi(u)=0$, Indiana Univ. Math. J. 30 (2) (1981) 161-177.

[4] J. Boussinesq, Theorie analytique de la chaleur, vol 2, Gauthier-Villars, Paris, 1903.

[5] J.1. Díaz, Resultados y métodos sobre la propiedad de extinción en tiempo finito para ecuaciones de evolución, Publ. Mat. 19 (1980) 93-115.

[6] J.I. Díaz, G. Galiano, On the Boussinesq System with nonlinear thermal diffusion, Proc. 2nd World Congress of Nonlinear Analysts, Athens, Greece, 10-17 July 1996, Elsevier, Amsterdam, 1997.

[7] J.I. Diaz, G. Galiano, Existence and uniqueness of solutions of the Boussinesq system with nonlinear thermal diffusion, Topological Methods Nonlinear Anal. (volume dedicated to Olga Ladyzhenskaya) (1999) to appear.

[8] J.I. Diaz, L. Veron, Local vanishing properties of solutions of elliptic and parabolic quasilinear equations, Trans. Amer. Math. Soc. 290 (2) (1985) 787-814. 
[9] J.I. Díaz, I.I. Vrabie, Compactness of the Green Operator of nonlinear diffusion equations: applications to Boussinesq type systems in Fluid Dynamics, Topological Methods Nonlinear Anal. 4 (1994) $399-416$.

[10] P.C. Fife, The Benard Problem for general fluid dynamical equations and remarks on the Boussinesq Approximation, Indiana Univ. Math. J. 20 (4) (1970) 303-326.

[11] C. Foias, O. Manley, R. Temam, Attractors for the Bénard Problem: existence and physical bounds on their fractal dimension, Nonlinear Anal. 11 (8) (1987) 939-967.

[12] G. Galiano, Sobre algunos problemas de la Mecánica de Medios Continuous en los que se originan Fronteras Libres, Tesis, Universidad Complutense de Madrid, 1997.

[13] G. Galiano, M.A. Peletier, Spatial localization for a general reaction-diffusion system, Ann. Fac. Sci. Toulouse (1999), to appear.

[14] O. Gontscharowa, About the uniqueness of the solution of the two-dimensional nonstationary problem for the equations of free convection with viscousity depending on temperature, Red Sib. Mat. J. V92 (260) (1990).

[15] D.D. Joseph, Stability of Fluid Motions I and II, Tracts in Natural Philosophy, vol 28, Springer, Berlin, 1976.

[16] J. Leray, Etude de diverses équations intégrales nonlinéaires et de quelques problemes que pose l'hydrodinamique, J. Math. Pures. Appl. 12 (1933) 1-82.

[17] J.M. Milhaljan, A rigorous exposition of the Boussinesq Approximations applicable to a thin layer of fluid, Astron. J. 136 (3) (1962) 1126-1133.

[18] A. Oberbeck, Uber die Wärmeleitung der Flussigkeiten bei der Berücksichtigung der Strömungen infolge von Temperaturdifferenzen, Ann. Physik Chemie 7 (1879) 271.

[19] J.F. Rodrigues, Weak solutions for thermoconvective flows of Boussinesq-Stefan type, in: J.F. Rodrigues, A. Sequeira (Eds.), Mathematical Topics in Fluid Mechanics, Pitman Research Notes in Mathematics Series, No. 274, Harlow, 1992, pp. 93-116.

[20] J. Rulla, Weak solutions to Stefan Problems with prescribed convection, SIAM J. Math. Anal. 18 (6) (1987) $1784-1800$.

[21] B. Straughan, The Energy Method, Stability and Nonlinear Convection, Springer, New York, 1992. 